\title{
Do DNA and Guanine Quench Fluorescence of Conjugated Cationic Polymers by Induced Aggregation?
}

\author{
Matthew L. Davies, ${ }^{1,2}$ Peter Douglas, ${ }^{1}$ Hugh D. Burrows, ${ }^{2, *}$ \\ M. Graça Miguel, ${ }^{2}$ Alastair Douglas ${ }^{3}$ \\ ${ }^{1}$ Chemistry Group, School of Engineering, Swansea University, \\ Singleton Park Swansea, SA2 8PP, UK \\ ${ }^{2}$ Departamento de Química da Universidade de Coimbra, Rua Larga, \\ 3004-535 Coimbra, Portugal \\ ${ }^{3}$ AD Technology Consulting Limited, Swansea, SA2 7UZ, UK
}

Received 14 May 2009; accepted 21 May 2009

\begin{abstract}
DNA and guanine are efficient fluorescence quenchers of the cationic conjugated polymer, poly $\{9,9$-bis[N,N-(trimethylammonium)hexyl] fluorene-co-1,4-phenylene $\}$ (CCP). Studies with CCPs, of average chain length $\sim 6,12$ and 100 repeat units, with single strand (ss) DNA, double strand (ds) DNA, and guanine, in 25/75 acetonitrile/water (v/v) mixtures result in Stern-Volmer quenching plots that show upward curvature. Initial Stern-Volmer constants, $\mathrm{k}_{\mathrm{SV}}$, are in the range $\approx 3-20 \times 10^{7} \mathrm{M}^{-1}$ which is much higher than possible by diffusional encounter quenching. Aggregation studies in acetonitrile/water mixtures show that aggregation is also an effective quencher of CCP fluorescence, and we note that both aggregation and quenching by DNA or guanine is accompanied by a reduction in solution absorbance at $380 \mathrm{~nm}$. Comparison of the relationship between changes in absorbance and changes in emission intensity suggest that both solvent and chemical induced fluorescence quenching are due to aggregation. We interpret the correlated changes in absorption and emission, high quenching constants, and upward curving Stern-Volmer plots as evidence that the dominant mechanism for fluorescence quenching by DNA or guanine is via induced aggregation of the polymer. The upward curvature of Stern- Volmer plots and high $\mathrm{k}_{\mathrm{SV}}$ values for DNA and guanine are indicative of "aggregate energy migration quenching" in which CCP aggregates around a DNA or guanine molecule to form an aggregate complex in which excitation energy migrates between and along the polymer chains until it is quenched at an aggregate trap.
\end{abstract}

Keywords: cationic conjugated polyelectrolytes, DNA, guanine, quenching, aggregation, energy migration.

\footnotetext{
* Corresponding author. E-mail address: burrows@ci.uc.pt
} 


\section{Introduction}

Cationic conjugated polyelectrolytes (CCPs) are currently the subject of a great deal of research due to their valuable optical and electronic properties [1-5]. There is also research on CCPs as potential DNA biosensors arising from the potential for electrostatic interactions with the phosphate groups of DNA [6,7]. In particular, emphasis has recently focussed on the potential of cationic conjugated polymers (CCPs) in sequence specific DNA assays [8-10]. Such sequence specific assays can be designed by combining the electrostatic interactions between cationic conjugated polymers and negatively charged DNA with appropriate recognition of base pairs, using, for example, protein nucleic acids (PNA), and resonance energy transfer [2,11].

In this study we examine the absorption and fluorescence properties of three fluorescent cationic conjugated polymers (CCPs), poly $\{9,9-b i s[N, N, N-$ (trimethylammonium)hexyl] fluorene-co-1,4-phenylene (Fig. 1) of varying chain lengths, in water/acetonitrile mixtures, and in the presence of DNA and guanine. The chemical structure of this CCP is useful as the responsive basis for biological and chemical detection designs based on optical properties since it allows for effective electronic coupling and thus fast intra- and interchain energy transfer, important because the polymer chain can act as a path for the transfer of excitation energy to energy acceptors and quenchers.

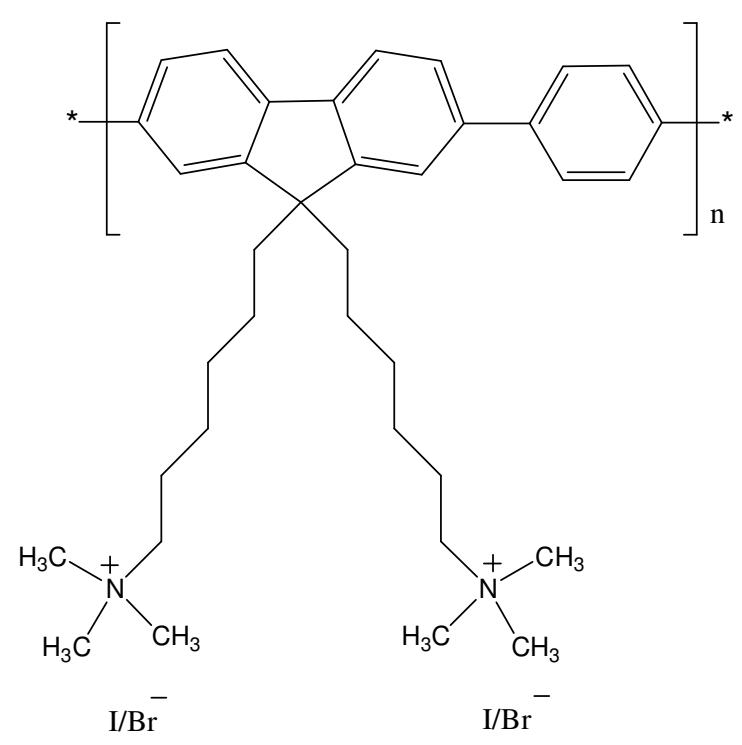

Figure 1. Structure of CCP 


\section{Experimental \\ Materials}

Bacteriophage T4 DNA (165.6 kilobase pairs, contour length of $57 \mu \mathrm{m}$ ) was purchased from Wako Nippon Gene. The three conjugated cationic polyelectrolytes $\quad\{9,9-b i s[N, N-(t r i m e t h y l a m m o n i u m) h e x y l] \quad$ fluorene-co-1,4phenylene (Fig 1), CCP-S (short), CCP-M (medium), and CCP-L (long) have average molecular weights, $M_{n}$ of $3.5,6.5$, and $52 \mathrm{~kg} \mathrm{~mol}^{-1}$ corresponding to average number of repeat units of 5.7, 12.4 and 99. Spectroscopic grade acetonitrile (Lab-Scan) and Millipore Milli-Q deionized water were used throughout.

\section{Methods}

Absorption measurements were made using $1 \mathrm{~cm}$ quartz cuvettes on a Shimadzu UV-2100 spectrophotometer. Fluorescence spectra were measured in $1 \mathrm{~cm}$ quartz cuvettes on a Jobin Yvon-Spex Fluorolog 3-22 instrument with $4.0 \mathrm{~nm}$ excitation and emission slits. Fluorescence spectra were corrected for the spectral response of the light source ( $450 \mathrm{~W}$ xenon lamp) and detector. The fluorescence quantum yields were measured against $\alpha$-4-oligothiophene $\left(\Phi_{\mathrm{f}}=0.16\right.$ in acetonitrile [12]), as standard using solutions of identical absorbance (0.37) with $379 \mathrm{~nm}$ excitation $[13,14]$. DNA concentrations used cover the range $0,1.25-12.4 \times 10^{-8} \mathrm{M}$ in base pairs. Single stranded DNA (ssDNA) was produced from the double stranded DNA (dsDNA) so that the base pair concentrations were kept the same. Guanine concentrations were ca. $1 / 5^{\text {th }}$ of DNA since the guanine content of T4 DNA is known to be $\sim 18 \%$ [15]. Stern-Volmer (SV) plots have been corrected for any changes in absorbance associated with change in solvent mixture or with the addition of DNA or guanine. Solvent mixtures are given as v/v.

\section{Results and discussion}

\section{Aggregation of CCPs in acetonitrile:water mixtures}

Figure 2a shows the absorption spectra for CCP-M in 25:75 and 1:99 acetonitrile:water. At low acetonitrile concentration the absorbance of the ca. 380 $\mathrm{nm}$ band $\left(\boldsymbol{\varepsilon}_{380}\right)$ is reduced, probably due to aggregation. The change in emission with increasing acetonitrile concentration for CCP-M in aqueous solution is shown in figure $2 \mathrm{~b}$. The emission intensity increases with acetonitrile concentration until ca. 25:75 acetonitrile/water after which there is no significant increase in intensity. Similar behaviour is also observed for CCP-S and CCP-L. We attribute both the increase in $\Phi_{\mathrm{f}}$ and increase in $\boldsymbol{\varepsilon}_{380}$ to the break up of polymer aggregates. It is thought that all the CCPs are still aggregated to some extent even in 25:75 acetonitrile:water. Since we wanted to use the minimum amount of acetonitrile that gave a high $\Phi_{\mathrm{f}}$ 25:75 acetonitrile/water was used as solvent in all the DNA quenching experiments. In this solvent mixture $\Phi_{\mathrm{f}}$ values are: $\mathrm{CCP}-\mathrm{S}=0.28, \mathrm{CCP}-$ $\mathrm{M}=0.34$ and $\mathrm{CCP}-\mathrm{L}=0.36$. 
a)

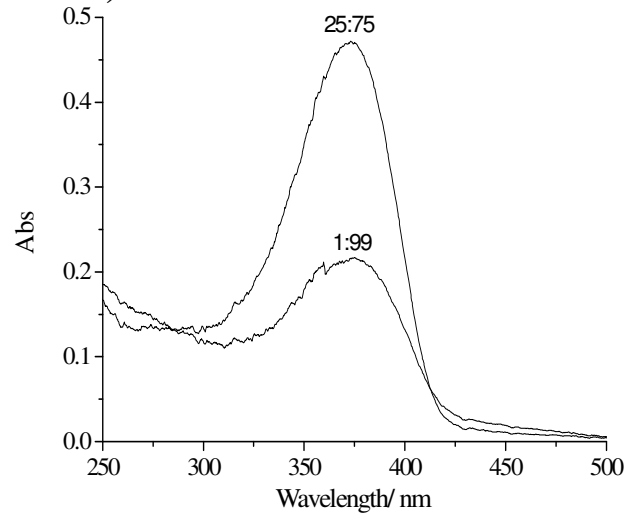

b)

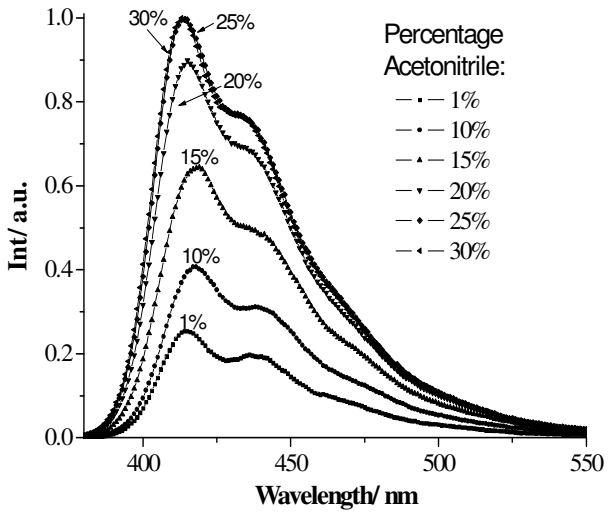

Figure 2. a) Absorption of CCP-M in 1:99 and 25:75 acetonitrile:water. b) Change in emission of CCP-M with solvent composition.

\section{Interaction of CCPs with DNA and guanine}

Addition of DNA or guanine to CCPs gives a decrease in both the absorption and emission intensity (Fig. 3).

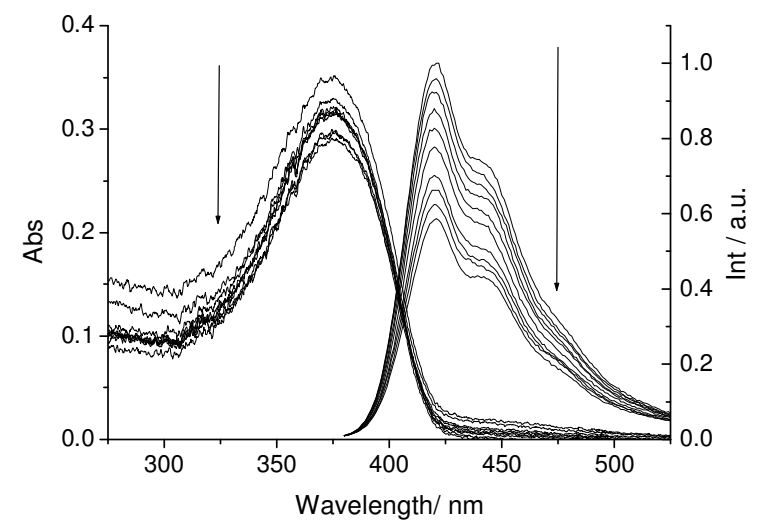

Figure 3. Change in absorption and emission of CCP-S with [dsDNA] increasing from 0 to $12.4 \times 10^{-8} \mathrm{M}$ base pairs.

Figure 4 shows typical Stern-Volmer plots; all show an upward curvature. Table 1 collects initial Stern-Volmer quenching constants $\left(\mathbf{K}_{\mathbf{S V}}\right)$ i.e. those for low quencher concentrations when the Stern-Volmer plots are reasonably linear. $\mathbf{K}_{\mathbf{S v}}$ values increase with $\mathrm{CCP}$ chain length. Given the short lifetime of CCP fluorescence $(\sim 500 \mathrm{ps})$ these quenching constants would give an apparent bimolecular quenching rate constant very much higher than the diffusion controlled limit for dynamic quenching. 


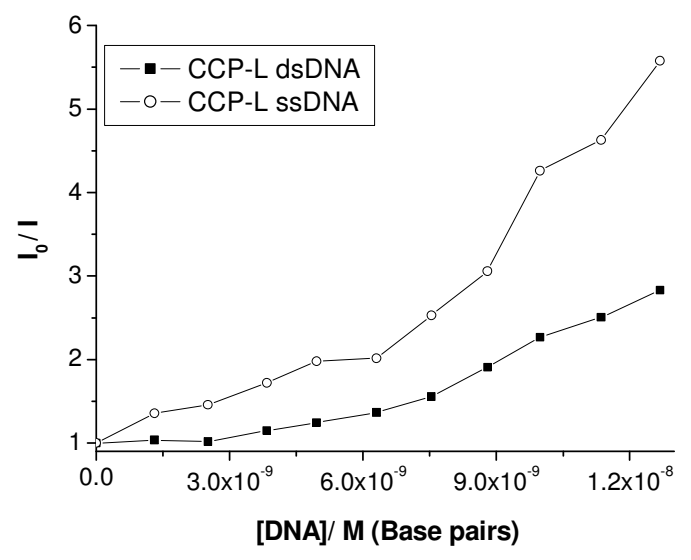

Figure 4. Stern-Volmer plots for quenching of CCP-L fluorescence by DNA.

Table 1. Initial Stern-Volmer quenching constants, $\mathbf{K}_{\mathrm{SV}} / \mathbf{1 0}^{\mathbf{7}} \mathbf{M}^{\mathbf{- 1}}$.

\begin{tabular}{|c|c|c|c|}
\hline Sample & Quencher & & \\
\hline & Guanine & dsDNA & ssDNA \\
\hline CCP-S & 2.8 & 2.9 & 4.3 \\
\hline CCP-M & 5.3 & 4.3 & 6.5 \\
\hline CCP-L & 8.8 & 9.6 & 18 \\
\hline
\end{tabular}

Addition of either DNA or guanine also results in a reduction in $\boldsymbol{\varepsilon}_{380}$ (figure 3). Recent fluorescence and atomic force microscopy studies indicate that DNA and CCP form structured aggregates in solution, and both the fluorescence quenching and changes in absorption caused by addition of DNA or guanine can be explained in this way [16]. Figure 5 shows a plot of the fractional change in emission intensity against fractional change in absorption intensity for CCP-L as either solvent mixture ( $\mathrm{I}_{\max }$ taken as that for 25:75 acetonitrile/water), or quencher concentration (with $\mathrm{I}_{\max }$ taken as that for no quencher present) are varied. The two sets of data lie on a single trend line, suggesting that for both solvent changes and DNA/guanine addition the changes in emission intensity are associated with changes in absorption intensity, irrespective of whether these absorption changes are brought about by varying solvent or quencher concentration. This suggests that the quenching processes are also the same, i.e. the dominant mechanism for fluorescence quenching by DNA or guanine is via induced aggregation of the polymer. Such a mechanism is supported by the observation that the amount of quenching is linearly dependent upon quencher concentration, which gives the upward curving Stern-Volmer plots, and which suggests that each active quenching unit acts as an independent centre around which aggregation of the polymer can occur. We suggest that the high quenching constants arise from "aggregate energy migration quenching" in which CCP 
alone, (in the case of solvent induced aggregation quenching), or $\mathrm{CCP}$ and DNA/guanine, (in the case of DNA or guanine aggregation quenching), form an aggregate complex in which excitation energy migrates between and along the polymer chains until it is quenched at an aggregate "trap".

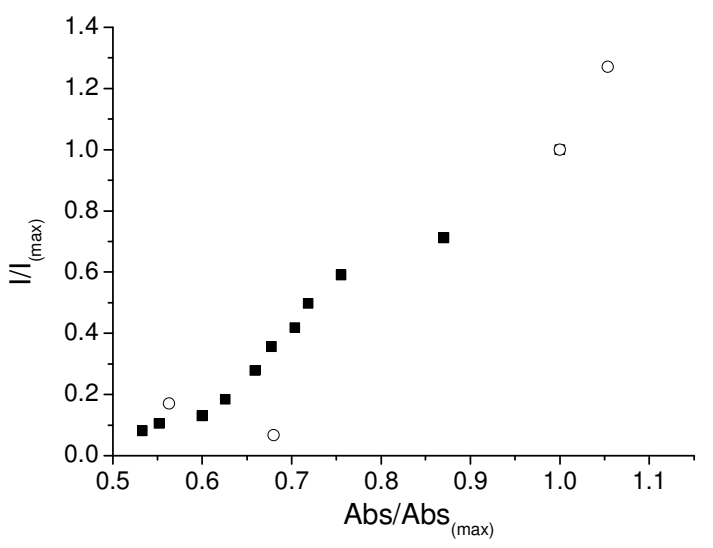

Figure 5. Ratio of emission against ratio of absorbance for CCP-L for changes in solvent composition (open circles ) and quencher concentration (filled squares).

\section{Conclusions}

Use of acetonitrile as co-solvent breaks up aggregates of CCPs formed in water. This is reflected by a $\sim 10$ fold increase in fluorescence quantum yield, and a ca. twofold increase in absorption extinction coefficient at $380 \mathrm{~nm}$. However, even at acetonitrile/water mixtures of ca. 25:75 there is evidence for aggregation. All CCPs are extremely sensitive to fluorescence quenching by dsDNA, ssDNA and guanine, with sensitivity increasing with chain length. In addition to quenching fluorescence, addition of these compounds also results in a reduction in absorption at $380 \mathrm{~nm}$. We interpret the correlated changes in absorption and emission, high quenching constants, and upward curving Stern-Volmer plots as evidence that the dominant mechanism for fluorescence quenching by DNA or guanine is via "aggregate energy migration quenching" in which DNA induces aggregation of the polymer to give an aggregate complex in which excitation energy migrates between and along the polymer chains until it is quenched at an aggregate trap. We are currently evaluating this idea using a program previously developed for energy migration in mixed 1-2D arrays [17].

\section{Acknowledgment}

We are grateful to Professor U. Scherf and Dr R. Mallavia for the CCPs used in this study. This work was funded by CIPSNAC (contract number: MRTN-CT-2003-504932), NEONUCLEI, Swansea University and POCI/FCT/FEDER. 


\section{References}

1. H.D. Burrows, V.M.M. Lobo, J. Pina, M.L. Ramos, J. Seixas de Melo, A.J.M. Valente, M.J. Tapia, S. Pradhan, and U. Scherf. Macromolecules 37 (2004) 7425-7427.

2. B. Liu, G.C. Bazan, Chem. Mater. 16 (2004) 4467-4476.

3. B.S. Gaylord, A.J. Heeger, G.C. Bazan, J. Am. Chem. Soc. 125 (2003) 896900.

4. S. Wang, B. Liu, B.S. Gaylord, G.C. Bazan, Adv. Funct. Mater. 13 (2003) 463-467.

5. L. Chen, X. Su, D. McBranch, D.J. Whitten, J. Am. Chem. Soc. 122 (2000) 9302-9303.

6. S.W. Thomas, G.D. Joly, T.M. Swager, Chem. Rev. 107 (2007) 1339-1386.

7. K.E. Achyuthan, T.S. Bergstedt, L. Chen, R.M. Jones, S. Kumaraswamy, S.A. Kushon, K.D. Ley, L. Lu, D. McBranch, H. Mukundan, F. Rininsland, X. Shi, W. Xia, D.G. Whitten, J. Mater. Chem. 15 (2005) 2648-2656.

8. H.A. Al-Attar, A.P. Monkman. Adv. Funct. Mater. 18 (2008) 2498-2509.

9. C. Chi, A. Mikhailovsky, G.C. Bazan, J. Am. Chem. Soc. 129 (2007) 1113411145.

10. K.Y. Pu, S.Y.H. Pan, B.J. Liu, Phys. Chem. B 112 (2008) 9295-9300.

11. C.V. Hoven, A. Garcia, G.C. Bazan and T.Q. Nguyen, Adv. Mater. 20 (2008) 3793-3810.

12. R.S. Becker, J. Seixas de Melo, A.L. Macanita and F. Elisei, J. Phys. Chem. 100 (48) (1996) 18683 - 18685.

13. A.T.R. Williams, S.A. Winfield, J.N. Miller, Analyst 108 (1983) 1067-1071.

14. J.N. Miller, In Standards in Fluorescence Spectrometry, Chapman and Hall: New York, 1981; p 75.

15. K. Yoshikawa, Y. Yoshikawa and T. Kanbe, Chem. Phys. Lett. 354 (2002) 354-359.

16. M.L. Davies, H.D. Burrows, M.C. Morán, M.G. Miguel and P. Douglas, Submitted to Biomacromolecules, 2009.

17. R.C. Evans, D. Ananias, A. Douglas, P. Douglas, L.D. Carlos, J. Rocha, J. Phys. Chem. C 112 (2008) 260-268. 